\title{
IATROGENIAS NO CUIDADO AO IDOSO NO CONTEXTO PANDEMIA COVID-19
}

\author{
Graziele Ribeiro Bitencourt' \\ ORCID: 0000-0002-9130-9307 \\ Carleara Weiss" \\ ORCID: 0000-0003-4421-0985
}

Patrícia de Fátima Augusto Barros'II ORCID: 0000-0003-4713-7576

Ana Carolina Siqueira de Carvalho'"I ORCID: 0000-0002-4222-127X

Cristiane dos Santos Rosalv ORCID: 0000-0002-6001-7053

'Universidade Federal do Rio de Janeiro-Campus Macaé. Macaé, Rio de Janeiro, Brasil.

"State University of New York at Buffalo. Buffalo, Nova Yorque, Estados Unidos.

"'Universidade Federal Fluminense. Niterói, Rio de Janeiro, Brasil.

IVILPI-Espaco Vianney. Niterói, Rio de Janeiro, Brasil.

Autor Correspondente: Graziele Ribeiro Bitencourt E-mail: grazielebitencourt@macae.ufrj.br

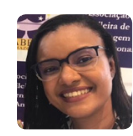

Como citar:

Bitencourt GR, Weiss C, Barros PFA, Carvalho ACS, Rosa CS. latrogenias no cuidado ao idoso no contexto pandemia covid-19. In: Santana RF (Org.). Enfermagem gerontologica no cuidado do idoso em tempos da COVID 19. 2.ed.rev. Brasilia, DF: Editora ABEn; 2020. p 89-94.

(Serie Enfermagem e Pandemias, 2). https://doi.org/10.51234/aben.20.e02.c14

\section{INTRODUÇÃO}

A palavra "iatrogenia" deriva do grego (iatros = médico / gignesthai $=$ nascer, que deriva da palavra genesis $=$ produzir). Trata-se da alteração patológica no paciente por práticas comumente evitáveis e impróprias de profissionais de saúde. Podem não ser intencionais, mas geralmente estão relacionadas ao desconhecimento das especificidades do processo de envelhecimento nas práticas de cuidado(1).

No contexto da pandemia da infecção pelo novo coronavírus (COVID-19), por se tratar de uma doença nova, ainda sem tratamento específico e com risco de contaminação dos profissionais, as estratégias de prevenção e controle estão em processo de desenvolvimento. Entretanto, algumas dessas precisam ser discutidas se cabem ou não no idoso. Refletir sobre a diferenciação dessa clientela ao adulto pode ser importante para a qualificação e segurança do cuidado prestado, principalmente, no contexto pandêmico em que há um panorama de medo e incertezas.

Idade avançada, número de comorbidades, complexidade das patologias, uso de múltiplos medicamentos, tempo de internação, gravidade da doença, limitações cognitivas e funcionais são alguns dos principais sinais de alerta nesse cenário(1). Do mesmo modo, essas variáveis são discutidas como comuns ao idoso com COVID-19 e relacionadas à piora do prognóstico, trazendo a necessidade de reflexão da vulnerabilidade dessa clientela associada tanto a doença quanto às estratégias terapêuticas estabelecidas(2).

Por outro lado, há escassez de estudos que abordem tanto como a COVID-19 se estabelece no idoso quanto sobre a efetividade das intervenções específicas. Isso aumenta a necessidade de reflexão da prática, no sentido de dar visibilidade aos principais riscos iatrogênicos e estimular novas abordagens de análise sobre como as respostas humanas se comportam nesta clientela.

Faz-se necessária, portanto, a expertise da enfermagem gerontológica para dar atenção às especificidades ao idoso 
e ao risco do cuidado inespecífico. Nesse sentido, como será que as iatrogenias podem ser estabelecidas no cuidado ao idoso no contexto da COVID-19?

\section{OBJETIVO}

Descrever as principais iatrogenias no cuidado ao idoso no contexto da pandemia COVID-19

\section{MÉTODO}

Trata-se de um estudo descritivo, tipo análise reflexiva da literatura, baseado em artigos científicos e normas técnicas publicadas de janeiro a junho de 2020.

\section{RESULTADOS}

No contexto da COVID-19 precisa-se refletir como as iatrogenias podem interferir no cuidado ao idoso nos cenários da prática, conforme a Figura 1.

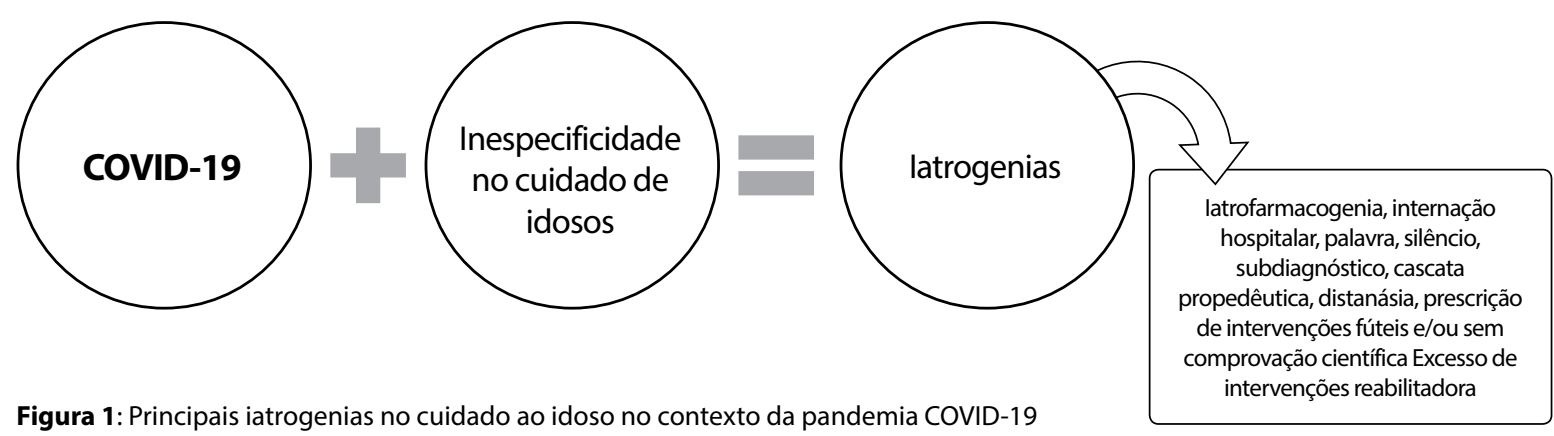

As principais situações que podem envolver a iatrogenia em idosos incluem iatrofarmacogenia, internação hospitalar, palavra, silêncio, subdiagnóstico, cascata propedêutica, distanásia e prescrição de intervenções fúteis (iatrogenias do cuidado) e/ou sem comprovação científica e excesso de intervenções reabilitadoras ${ }^{(3)}$.

\section{latrofarmacogenia}

Relaciona-se ao uso de medicamentos inespecíficos, polifarmácia, interação medicamentosa e do desconhecimento das alterações farmacocinéticas e farmacodinâmicas associadas ao envelhecimento(3).

A polifarmácia contribui para distúrbios do sono em idosos em ambientes hospitalares, domiciliares e instituições de longa permanência ${ }^{(4)}$. Alterações naturais do sono do idoso, típicas do envelhecimento, tais como redução na duração do sono em horas, são potencialmente exacerbadas pela iatrofarmacogenia. De modo geral, as alterações fisiológicas do sono do idoso produzem efeitos psicossociais ${ }^{(5)}$.

Neste contexto, a iatrofarmacogenia, somada às alterações fisiológicas do sono, prejudicam a qualidade de vida do idoso. Como consequência, observa-se insônia, confusão mental, alterações no comportamento e na memória e prejuízo a qualidade de vida do idoso.Benzodiazepínicos se destacam como agentes relacionados com interação medicamentosa e distúrbios de sono no idoso. ${ }^{(5)}$.

O tratamento da COVID-19 potencializa o risco para iatrofarmacogenia em idosos, tendo em vista a combinação de medicamentos necessária para combater o coronavírus SARS-COV-2. Enfermeiros devem identificar precocemente a iatrofarmacogenia, promover a priorização de um plano seguro, bem como a reorganização gradual do plano terapêutico. 


\section{Internação hospitalar}

Os casos graves da COVID-19 têm sido associados à necessidade de internação hospitalar prolongada para alívio de sinais e sintomas graves como o desconforto respiratório. Devido ao risco de rápida progressão da doença, os idosos não são tratados especificamente na atenção básica e são comumente submetidos a internações por conta da COVID-19. Destaca-se que a infecção pelo SARS-CoV-2 é alta no cenário hospitalar, com taxas de mortalidade para indivíduos maiores de 80 anos de até $15 \%{ }^{(6)}$.

Entretanto, essa internação pode aumentar a dependência e trazer outras complicações para a clientela idosa. Dentre essas há a desnutrição, depressão, quedas, estados confusionais, infecções e diminuição da mobilidade ${ }^{(7)}$. Além disso, ainda podem ser observadas outras iatrogenias como a cascata propedêutica, prescrição de intervenções fúteis e/ou sem comprovação científica e distanásia.

\section{latrogenia da palavra}

O ato de informar refere-se ao momento inicial de revelação da doença, tratamento ou prognóstico e comunicar está inserido no processo relacional de interação entre o paciente, a família e o profissional de saúde. Nesse sentido a comunicação de más ou difíceis notícias atravessa o enfrentamento em diversos estágios pelo próprio paciente e família e está inserido como uma iatrogenia ${ }^{(3)}$.

As dificuldades de comunicação podem acontecer em qualquer situação relacional, especialmente em discussões nas quais estejam envolvidas questões de doença e morte. Por se tratar de uma doença nova, ainda sem tratamento ou vacina e com pior prognóstico no idoso, a COVID-19 pode gerar interfaces importantes na comunicação com o paciente e a família, tanto na divulgação do diagnóstico quanto da impossibilidade de tratamento. Há possibilidade de limitações no enfrentamento de ordem pessoal do paciente e pela falta de reflexão ou preparo do profissional para realização dessa tarefa devido ao desconhecimento de técnicas na abordagem da doença com o paciente e família ${ }^{(7)}$.

\section{latrogenia do silêncio}

Comunicar com qualidade passa pela compreensão do que queremos trocar com os pacientes e pela união da capacidade de envolvimento com o outro, a fim de estabelecer a relação de ajuda, disponibilidade e conhecimento. Quanto essa atividade não acontece e decorre a dificuldade de ouvir adequadamente o paciente e sua família é estabelecida a iatrogenia do silêncio(3).

A COVID-19 implicou no desdobramento do profissional em estabelecer comunicações mais rápidas com pacientes e familiares visando diminuir o tempo de contato e o risco de contaminação. Em contrapartida, o idoso pode precisar de mais tempo para articular o pensamento e se expressar. Associado a necessidade de isolamento social, o tempo de contato e as suas oportunidades de escuta do idoso diminuem, o que aumenta o risco desta iatrogenia ${ }^{(7)}$.

Novas estratégias de comunicação como o uso de tecnologias podem ser uma alternativa, mas precisam ser efetivas, de modo a oportunizar o espaço requerido ao cuidado, dar o tempo necessário às avaliações, envolver as especificidades do idoso, sem expor os profissionais e o a pessoa idosa.

\section{Subdiagnóstico}

Esta iatrogenia refere-se a tendência em atribuir as queixas do idoso à idade ${ }^{(3)}$. No contexto da COVID-19, profissionais com experiência gerontológica geralmente conhecem as apresentações atípicas de doenças e distúrbios clínicos comuns no idoso. Entretanto, o primeiro atendimento nem sempre é realizado por esses profissionais com maior expertise, o que pode levar ao subdiagnóstico. 
Os achados típicos em pacientes com COVID-19 são febre, tosse e dispnéia. Em um relatório inicial de 138 pacientes em Wuhan, China, com idade média de 56 anos (22-92 anos), a maioria sem múltiplas comorbidades, febre foi identificada em $99 \%$, fadiga em $70 \%$, tosse em $59 \%$, anorexia em $40 \%$, mialgia em $35 \%$ e dispnéia em $31 \%$. No entanto, em idosos, especialmente os mais frágeis e com múltiplas condições crônicas, podem ser afebris e não apresentar tosse, desconforto no peito ou produção de escarro, o que pode conduzir ao subdiagnóstico da doença. Taquipnéia, estado mental alterado ou delirium e taquicardia inexplicada ou diminuição da pressão arterial podem ser as manifestações clínicas presentes. Há registros ainda de início do quadro com mal-estar, dores musculares, febre baixa e tosse que evoluíram para dificuldade respiratória na segunda semana da doença. A febre não era proeminente em vários $\operatorname{casos}^{(2)}$.

Presença de demência, histórico de acidente vascular cerebral ou outros problemas de saúde podem mascarar as manifestações da infecção por COVID-19. Portanto, qualquer alteração significativa no estado clínico em relação que não tenha explicação imediata pode ser causada por infecção ou sepse e deve ser avaliada como COVID-19 durante a atual epidemia, evitando assim o subdiagnóstico.

\section{Cascata propedêutica}

Trata-se de solicitações de exames de forma desnecessária, extensiva e sem justificativa ou indicação precisa( ${ }^{(3)}$. No contexto da COVID-19, com protocolos ainda em construção, a tendência de solicitações pouco coesas pode expor ainda mais o idoso. Isso porque a estratificação por idade, considerando a especificidade de cada uma delas ainda é um processo que carece de construção, principalmente em doenças ainda pouco conhecidas (2).

É necessário que cada profissional considere as características do processo de envelhecimento em suas intervenções, como apresentação dos sinais e sintomas e consideração de outros quadros clínicos para solicitação de exames com mais assertividade.

\section{Distanásia}

Considerada como o prolongamento artificial da vida sem perspectiva de reversibilidade, com sofrimento para o paciente e sua família ${ }^{(3)}$, a distanásia requer uma ampla reflexão quando associada ao envelhecimento.

As ações tomadas para enfrentamento da pandemia são de ordem emergencial, e, por sua natureza, tem por objetivo a proteção da coletividade no que tange a saúde pública. Por tal motivo a Lei 13.979/2020 autoriza, por exemplo, a realização compulsória de testagem, de vacinação, exumação de corpos, sujeitando os indivíduos que se negarem a atender às normas sanitárias à responsabilização por seus atos ${ }^{(8)}$.

Observa-se que o caráter personalizado da atenção em saúde, tão necessário para respeito aos direitos humanos dos pacientes, foi colocado em segundo plano. Tal excepcionalidade possui um alto custo para os idosos institucionalizados, como, por exemplo, para os que necessitam da assistência concomitante de seus familiares em instituições com carência de profissionais de cuidado ou ainda junto a pacientes em cuidados paliativos, o que propiciou o abandono dos idosos na doença e na morte ${ }^{(9)}$.

É necessário que sejam discutidas estratégias para tomadas de decisão sobre até que ponto há possibilidade de vida com qualidade após a COVID-19 ou quando as práticas de conforto podem ser a melhor alternativa. Práticas de cuidado durante a pandemia vêm sendo estudadas no mundo inteiro no intuito de determinar como o enfrentamento tem que ser realizado ou saber os malefícios de tais práticas, isso tem desafiado profissionais dos mais variados saberes. Entretanto, são medidas necessárias à compreensão dos conceitos de fragilidade e finitude, tão amplamente abordados quando remetidos a gerontologia.

\section{latrogenia dos cuidado}

Relacionadas às intervenções que impõem ao idoso ao risco desnecessário, como de prescrição de intervenções sem comprovação científica ${ }^{(3)}$. Em virtude da pandemia, os cenários tornaram-se mais exaustivos e 
superlotados. A demanda por cuidados e leitos é ainda maior do que o visto anteriormente. Nesse sentido, há uma lacuna em apontar para as possíveis iatrogenias por intervenções sem embasamento ao paciente idoso no contexto do COVID-19, como por exemplo, a contenção mecânica e uso de fraldas.

Embora essas medidas partam da premissa de evitar a circulação e contaminação de profissionais ou outros pacientes como por exemplo a diminuição dos acompanhantes, estas também podem impor ao idoso dependência e perda de autonomia. Como recomendações, devem-se instituir orientações para o comportamento desafiador em detrimento do uso da contenção mecânica. Podem ser incluídas nessa perspectiva a terapia de orientação para realidade de forma a assegurar o cuidado de enfermagem reabilitante, focado no estímulo à potencialidade ainda existente em cada sujeito, e na liberdade humana ${ }^{(10)}$.

As fraldas também podem se inserir nesse contexto iatrogênico na pandemia COVID-19 pelo paradoxo entre o potencial risco de contaminação e a indicação segura do uso, restrita aos casos graves de imobilidade, déficit cognitivo e incontinências. Desta forma, a análise da prática em idosos mostra-se como assistemática e, sem consideração dos critérios e o expõe a como incontinência urinária e fecal, lesões de pele e infecções ${ }^{(11)}$. Por outro lado, já foi identificada a presença do vírus nas fezes e urina, de modo que o maior número de trocas pode expor o profissional ainda mais a contaminação. Entretanto, esse menor número de trocas expõe o paciente ainda mais a complicações clínicas. Refletindo sobre esse uso na própria equipe de enfermagem, os profissionais ficam expostos aos mesmos ricos do uso de fraldas do paciente.

A recuperação do idoso pode ser mais eficaz se minimizados fatores associados a iatrogenias geradas pelos próprios profissionais de saúde. Para isso cabe cada membro da equipe de enfermagem avaliar as suas práticas e os riscos delas considerando os aspectos inerentes ao envelhecimento.

\section{latrogenia do excesso de intervenções reabilitadoras}

Trata-se do excesso de "equipe interdisciplinar"(3). Compreender as potencialidades e fragilidades do idoso é a primeira etapa do cuidado, de modo a considerar alguns limites do processo de envelhecimento no estabelecimento das metas e prioridades na assistência ao paciente.

Na pandemia COVID-19, esse é um dos desafios, no sentido de identificar quais são as fragilidades preexistentes do idoso, o que pode melhorar ou permanecer no estado inicial para garantir que um cuidado seja efetivo ${ }^{(2)}$. Essa premissa é importante para classificar quais são possibilidades de resultados do idoso e evitar o excesso de intervenções reabilitadoras. Um paciente, por exemplo, com dependência parcial para o autocuidado antes da COVID-19, pode ser estabelecido como meta permanecer desta forma e não evoluir para uma dependência total. Daí a importância da consideração dos princípios gerontológicos na prescrição de intervenções e estabelecimento de metas.

\section{Limitações do Estudo}

Há poucos estudos que abordam o idoso e iatrogenias no contexto da COVID-19, principalmente, sobre a identificação e análise da efetividade das intervenções e suas considerações nas especificidades no processo de envelhecimento.

\section{Contribuições para a Área}

Compreender como cuidar do paciente com COVID-19 vem sendo um desafio para a enfermagem, ainda maior no idoso tanto pela falta de estudos que abordem a temática quanto de profissionais qualificados nessa assistência. Portanto, refletir sobre a implicação do uso dos princípios gerontológicos na prescrição de cuidados e definições de metas precisa ser uma medida contínua para evitar iatrogenias e prejuízos ainda maiores nessa clientela. 


\section{CONCLUSÃO}

A prescrição de intervenções e estabelecimento de metas específicas ao idoso e detrimento da adaptação advindas da aplicabilidade ao adulto, precisam ser o principal foco do cuidado em gerontologia no contexto da COVID-19.

A partir disso, nesse estudo foi possível refletir sobre o cuidado ao idoso no contexto da pandemia. A lacuna nesta prática expõe essa clientela a iatrogenias, como iatrofarmacogenia, internação hospitalar, palavra, silêncio, subdiagnóstico, cascata propedêutica, distanásia e prescrição de intervenções fúteis e/ou sem comprovação científica e excesso de intervenções reabilitadoras.

\section{AGRADECIMENTO}

\section{Ao Departamento Científico de Enfermagem Gerontológica da ABEn Nacional.}

\section{REFERÊNCIAS}

1. Paraná. Secretaria de Estado da Saúde do Paraná. Superintendência de Atenção à Saúde. Linha guia da saúde do idoso. 2 ed. Curitiba: SESA, 2018. 126 p.

2. D'Adamo H, Yoshikawa T, Ouslander JG. Coronavirus Disease 2019 in Geriatrics and long-term care: the ABCDs of COVID-19. J Am Geriatr Soc. 2020;68(suppl 5):912-917. doi:10.1111/jgs.16445

3. Moraes EN, Azevedo RS. Fundamentos do cuidado ao idoso frágil. Belo Horizonte: Folium; 2016.

4. Kumar S, Wong PS, Hasan SS, Kairuz T. The relationship between sleep quality, inappropriate medication use, and frailty among older adults in aged care homes in Malaysia. PIOS one. 2019;14(suppl 10): e0224122. doi: doi.org/10.1371/journal. pone.0224122

5. Dean GE, Weiss C, Morris JL, Chasens ER. Impaired Sleep: A Multifaceted Geriatric Syndrome. Nurs Clin North Am. 2017;52(3):387-404. doi:10.1016/j.cnur.2017.04.009

6. Centers For Disease Control And Prevention. [Internet]. 2020. [Acesso em 16 de junho de 2020]. Disponível em: https:// www.cdc.gov/coronavirus/2019-nCoV/hcp/infection-control.html

7. Taylor LJ, Johnson SK, Nabozny MJ. Barriers to goal-concordant care for older patients with acute surgical illness: communication patterns extrinsic to decision aids. Ann Surg. 2018;267(suppl 4):677-82. doi:10.1097/ SLA.0000000000002282

8. Brasil. LEI No 13.979, de 6 de fevereiro de 2020. Diário Oficial da União 6 fev; 27(1 sup):1

9. Peisah C, Byrnes A, Doron II, Dark M, Quinn G. Advocacy for the human rights of older people in the COVID pandemic and beyond: a call to mental health professionals. Int Psychogeriatr. 2020;1-13. doi:10.1017/S1041610220001076.

10. Delvalle, R. et al . Contenção mecânica em instituição de Longa Permanência para Idosos: estudo transversal. Rev. Bras. Enferm. 2020; 73(3): e20190509. DOI: https://doi.org/10.1590/0034-7167-2019-0509.

11. Bitencourt GR, Alves LAF, Santana RF. Practice of use of diapers in hospitalized adults and elderly: cross-sectional study. Rev Bras Enferm. 2018;71(2):343-9. DOI: http://dx.doi.org/10.1590/0034-7167-2016-0341 